\title{
Bubble Dynamics in Nucleate Pool Boiling on Thin Wires in Microgravity
}

\author{
J. F. Zhao • G. Liu • S. X. Wan • N. Yan
}

Received: 30 December 2006 / Accepted: 27 November 2007 / Published online: 28 March 2008

(C) Springer Science + Business Media B.V. 2008

\begin{abstract}
A temperature-controlled pool boiling (TCPB) device has been developed to study the bubble behavior and heat transfer in pool boiling phenomenon both in normal gravity and in microgravity. A thin platinum wire of $60 \mu \mathrm{m}$ in diameter and $30 \mathrm{~mm}$ in length is simultaneously used as heater and thermometer. The fluid is R113 at $0.1 \mathrm{MPa}$ and subcooled by $26^{\circ} \mathrm{C}$ nominally for all cases. Three modes of heat transfer, namely single-phase natural convection, nucleate boiling, and two-mode transition boiling, are observed in the experiment both in microgravity aboard the 22nd Chinese recoverable satellite and in normal gravity on the ground before and after the space flight. Dynamic behaviors of vapor bubbles observed in these experiments are reported and analyzed in the present paper. In the regime of fully developed nucleate boiling, the interface oscillation due to coalescence of adjacent tiny bubbles is the primary reason of the departure of bubbles in microgravity. On the contrary, in the discrete bubble regime, it's observed that there exist three critical bubble diameters in microgravity, dividing the whole range of the observed bubbles into four regimes. Firstly, tiny bubbles are continually forming and growing on the heating surface before departing slowly from the wire when their sizes exceed some value of the order of $10^{-1} \mathrm{~mm}$. The bigger bubbles with about several millimeters in diameter stay on the wire, oscillate along the wire, and coalesce with adjacent bubbles. The biggest bubble with diameter of the order of $10 \mathrm{~mm}$, which was formed immediately after the onset of boiling, stays continuously
\end{abstract}

\footnotetext{
J. F. Zhao $(\bowtie) \cdot$ G. Liu $\cdot$ S. X. Wan $\cdot$ N. Yan

National Microgravity Laboratory, Institute of Mechanics,

Chinese Academy of Sciences,

15 Beisihuan Xilu,

Beijing 100080, China

e-mail: jfzhao@imech.ac.cn
}

on the wire and swallows continually up adjacent small bubbles until its size exceeds another critical value. The same behavior of tiny bubbles can also be observed in normal gravity, while the others are observed only in microgravity. Considering the Marangoni effect, a mechanistic model about bubble departure is presented to reveal the mechanism underlying this phenomenon. The predictions are qualitatively consistent with the experimental observations.

Keywords Bubble dynamics - Nucleate boiling . Microgravity · Marangoni effect

\section{Introduction}

Pool boiling in microgravity has become an increasing significant subject for investigation, since many potential applications exist in space and in planetary neighbors due to its high efficiency. However, the investigation in microgravity suffers for unique and stringent constraints in terms of size, power and weight of experimental apparatuses, and of number and duration of the experiments. Thus, only a partial and in some aspects contradictory knowledge of microgravity boiling has been attained so far. On the progress in this field, several comprehensive reviews are available. For example, Straub (2001) issued a comprehensive review of his own activity on this field from the early 1980s to date, while Di Marco (2003), Kim (2003), and Ohta (2003) recently issued reviews of microgravity boiling researches in Europe, in US, and in Japan, respectively. Thus, only results related to the present study, namely those on nucleate pool boiling on wires and cylinders in microgravity can shortly summarized in the following.

Using a $2.5 \mathrm{~m}$-high drop tower, Siegel and Usiskin (1959) carried firstly out the experimental studies on pool boiling on 
wires in microgravity. They found that heat transfer is enhanced in reduced gravity $\left(0.014 g_{0}\right.$, here $g_{0}$ denotes the terrestrial gravity) for pool boiling on a horizontal $0.5 \mathrm{~mm}$ dia. wire in water and alcohol, and that the enhancement decreases with the increase of heat flux. No influence of gravity was observed for boiling on a horizontal $0.5 \mathrm{~mm}$-dia. wire in $60 \%$ sucrose solution.

Tokura et al. (1995) performed experiments in JAMIC dropshaft on pool boiling of methanol on thin platinum wires (0.1 and $0.05 \mathrm{~mm}$ diameter). Two bubble patterns were reported. In the first pattern, relatively small bubbles sprung out from the wire and quasi-steady-state nucleate boiling in low heat flux conditions was obtained. The heat transfer coefficient was quite close to that in normal gravity. On the contrary, in the second one the bubbles coalesced laterally along the wire to eventually form large spherical bubble that enclosed the wire without detaching. Inside large bubble, the wire glowed white. The authors concluded that it is not possible to attain steady state boiling at high heat flux in microgravity. Coexistence of the two patterns was also observed.

Similar phenomenon was also observed by Motoya et al. (1999) in the pool boiling experiments of water on a bare platinum wire $(0.2 \mathrm{~mm}$ diameter) in JAMIC dropshaft, with a large coalescing bubble causing burnout at high heat flux. The pool boiling performance in microgravity was almost the same as in normal gravity, with reduction in CHF. The effect of scale deposition (calcium carbonate) on the wire was also studies. Modest degradation was found and the bubbles kept on detaching even at high heat flux from the scale wire.

Sitter et al. (1998) studied the effect of acoustic fields during nucleate boiling on a $0.25 \mathrm{~mm}$-dia. platinum wire in FC-72 using a 2.1s drop tower. Without acoustic field, decrease of heat transfer was evidenced. The heat transfer for a given wall superheat was always higher with the highintensity acoustic field than without, and the highest heat transfer occurred with the heater placed at the antinode for both terrestrial and microgravity boiling. Acoustic streaming was observed to bring colder ambient liquid to the wire, enhancing heat transfer.

Much extensive experiments on pool boiling on wires (0.05, 0.2 and $8 \mathrm{~mm}$ diameter) were performed by Straub and coworkers (Straub 2001; Straub et al. 1992; Straub and Micko 1996; Steinbichler et al. 1998) with various refrigerants (R113, R12, R134a) in parabolic flights, sounding rockets and orbital flights. For nucleate pool boiling on wires, the heat transfer was found to be almost independent of gravity level or slightly enhanced in microgravity. For the bubble dynamics observed in their experiment on nucleate pool boiling during a GAS flight, Straub reported the "cylindrically symmetrical" and the "two-dimensional plane" bubble behaviors, while no difference could be observed in the heat transfer values between them. The departure and bubble motion were also observed, which were caused by continuous coalescence processes. The departure diameters from wires and cylinder in their KC-135 experiments are as about four times of those in normal gravity, while a factor of $1.2 \sim 1.5$ is observed in the GAS Space Shuttle experiments, much smaller than the predictions of the common used correlations, such as Fritz (1934). They also reported their CHF data which were consistently lower than the corresponding terrestrial values, and could be successfully correlated in Lienhard and Dhir form (1973) for values of $R^{\prime}=$ $R \sqrt{g\left(\rho_{\mathrm{L}}-\rho_{\mathrm{G}}\right) / \sigma}$ as low as $5 \times 10^{-4}$. Furthermore, influence of liquid subcooling has also been studied, and various modes, particularly for bubble behaviors, are observed according to the degree of subcooling. Under subcooled conditions only, thermocapillary flow or Marangoni convection was observed. This leads the author to the hypothesis that the origin of thermocapillary flow is induced by the accumulation of inert gases.

Shatto and Peterson (1999) studied CHF using a cylindrical cartridge heater $(9.4 \mathrm{~mm}$ diameter $)$ immersed in slightly subcooled water at about $1 / 4$ atm aboard the NASA KC-135 airplane. In their study, $R^{\prime}<0.4$, indicating that the cylinder was a small one. The values of CHF increased with gravity during the nominally "zero-g" parabola, and were lower than the predictions by the correlation of Lienhard and Dhir (1973) by an average of $40 \%$. They argued that this difference is caused by thermocapillary effect. Following the work of McGillis and Carry (1996), they derived an semi-empirical correlation with an adjustable constant, which gave a good agreement with their data. An assumption that the temperature distribution along the bubble surface is not affected by the phase change is implied in the derivation of the correlation, which is different from Straub (2001).

Di Marco and Grassi (2002), Di Marco et al. (2001) conducted experiments of pool boiling of R113 and FC-72 on a $0.2 \mathrm{~mm}$-dia. platinum wire both in parabolic flight and in sounding rocket, with and without electric field. Data at enhanced gravity and at Martian gravity level $\left(0.4 g_{0}\right)$ were also collected. Despite a very evident change in bubble behavior, no influence of gravity level was found on the heat transfer coefficient in nucleate boiling on a wire without electric field. CHF was found to be reduced of about $50 \%$ in low gravity. The trend of the scaling of CHF, as the authors stated, is in conflict with others in the region of small Bond number or dimensionless wire radius $R^{\prime}$. The imposition of a cylindrical electrostatic field was found to have no significant effect on nucleate boiling heat transfer coefficient, but to be effective in reducing bubble size and increasing $\mathrm{CHF}$ in microgravity. 
Recently, using the same experimental facility as the present study, Zhao et al. (2005) performed some preliminary experiments on pool boiling in microgravity in the Drop Tower Beijing, which can provide a 3.6-s period of microgravity. The working fluid is R113 with medium subcooling $\left(\Delta T_{\text {sub }}=24^{\circ} \mathrm{C}\right)$. For nucleate boiling, the heat transfer was slightly enhanced. No change was observed for the bubble departure diameter in different gravity conditions. Lateral oscillation along the wire was always observed, which can lead to the lateral coalescence between adjacent bubbles and then the detachment of the new bubble. Sometimes, the new bubble formed by the lateral coalescence cannot depart from the wire immediately. It may enclose the wire and a bright spot will appear there. However, it cannot last long period and the boiling continues as nucleate boiling. For two-mode transition boiling, about $20 \%$ decrease of the heat flux was obtained, although the part of film boiling was receded in microgravity. The surface tension reforms the vapor film to a large spherical bubble enclosing the wire immediately after the release, and the part of film boiling will recede due to insufficiency of vapor. The wire glowed red inside the large bubble.

In brief, there are many experimental data obtained in the past years, and great advances have been made in the field of pool boiling heat transfer in microgravity. However, due to the high cost and low availability of flight opportunities, and to their limitations in space, time and power supply, a final assessment has yet to be completed, and some results are still controversial. The present work is a part of a research project on the pool boiling on thin wires in microgravity. A series of experiments both in microgravity aboard the 22nd Chinese recoverable satellite and in normal gravity on the ground before and after the space flight have been conducted. New sets of experimental data were obtained. In the present paper, dynamic behaviors of vapor bubbles observed in these experiments are analyzed, while other aspects will be reported elsewhere.

\section{Experimental Facility}

A temperature-controlled pool boiling (TCPB) device has been developed to study the bubble behavior and heat transfer in such phenomenon both in normal gravity on the ground and in microgravity aboard the 22nd Chinese recoverable satellite. Detailed description of the TCPB device (Fig. 1) can be found in Wan et al. (2003) Therefore, only a simple description will be presented briefly here.

A platinum wire of $60 \mu \mathrm{m}$ in diameter and $30 \mathrm{~mm}$ in length is simultaneously used as heaters and thermometers, with the advantage that because of its low thermal capacity, it reacted almost without any delay on changes in temperature and heat transfer, respectively. The ends of the wire are soldered with copper poles of $3 \mathrm{~mm}$ in diameter to provide a firm support for the wire heater and low resistance paths for the electric current. The heater resistance, and thus the heater temperature, is kept constant by a feedback circuit, which is similar to that used in constant-temperature hot-wire anemometry and that in constant-temperature microheaters by Rule and Kim (1999). Its adjustment is controlled by varying the resistance of the changeable resistance network. This resistance network comprises 16 parallel resistors and a 16-channel analog switch, which is controlled by a SCM (single-chip-microcomputer). Thus, 16 set-points of heater temperature can be obtained within a range of $35 \sim 135^{\circ} \mathrm{C}$, and most of them are located in the nucleate boiling range. A special procedure is used for the heater temperature: firstly, the stepped heater temperature is controlled upwards
Fig. 1 Diagram of boiling chamber and its accessories

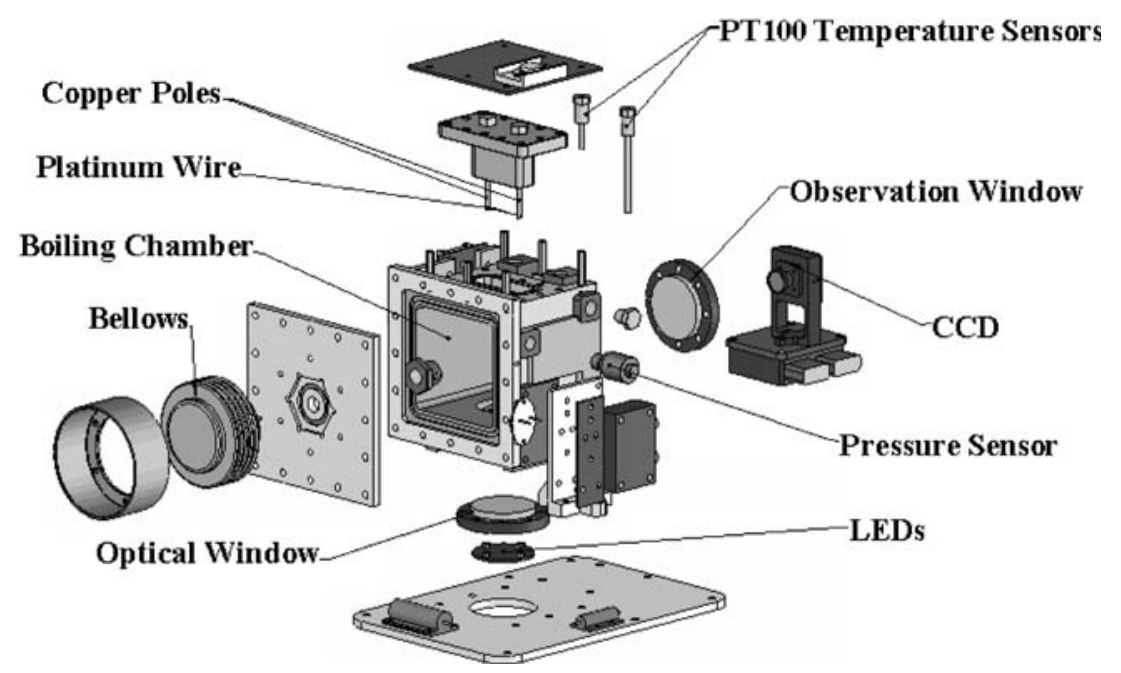


from the lowest one to the highest one, then downwards to the lowest one, and finally upwards again to the highest one. Furthermore, according to Straub (2001), each step lasts about $30 \mathrm{~s}$ in order to obtain steady pool boiling. In actual fact, except for those corresponding to the onset of boiling and the critical heat flux (CHF) in microgravity, the steady state can be achieved within $3 \mathrm{~s}$ for each step both in normal gravity and in microgravity. For the two exceptions, the steady state can also be achieved and last for about $10 \mathrm{~s}$.

The boiling chamber with a inner dimension of $80 \times 80 \times$ $95 \mathrm{~mm}^{3}$ is fixed inside an air-proof container. A bellows connected with the chamber will allow the pressure in the chamber to be approximately constant. Two platinum resistance thermometers with a range of $0 \sim 60^{\circ} \mathrm{C}$ are used to measure the bulk temperature of the fluid in the boiling chamber, which are calibrated to within $0.25^{\circ} \mathrm{C}$. The absolute pressure within the boiling chamber is measured using a pressure transducer with a range of $0 \sim 0.2 \mathrm{MPa}$ and an accuracy of $0.25 \%$ FS (full scale). Two LEDs (lightemitting diode) are used to light the boiling chamber through a diffusion window at the chamber bottom. A CCD video camera (WAT-660D, $537(H) \times 505(V)$ pixels) is used to obtain images of the motion of vapor bubble or film around the heater, which is recorded by a VCR at a speed of 25 fps. The voltages across the heater and a reference resistance, which is used to measure the electric current through the heater, are sampled at a rate of $20 \mathrm{~Hz}$ in space experiment, while a much higher sampling rate of $1,000 \mathrm{~Hz}$ is used in the ground experiments before and after the space flight. The analysis shows that the uncertainty of the heater temperature can be significantly reduced by using high sample rate (less than $3^{\circ} \mathrm{C}$ ), while the influence of the sample rate on the uncertainty of heat flux is negligible. The uncertainty of heat flux is less than $24 \mathrm{~kW} / \mathrm{m}^{2}$ in space experiment and $21 \mathrm{~kW} / \mathrm{m}^{2}$ in ground experiments, respectively. A lower sampling rate of $1 / 3 \mathrm{~Hz}$ is used for the outputs of the pressure transducer and the platinum resistance thermometers both in space and on the ground.

The test liquid is R113 of nominal $700 \mathrm{ml}$. A special procedure, which is repeated more than ten times over a 2day period, is adopted to remove dissolved gases: heating the liquid near to its boiling point, pulling the discharged gases out, and then cooling the liquid down.

Table 1 Conditions for the experiments on the ground and in space

\begin{tabular}{lccc}
\hline & System pressure & $\begin{array}{l}\text { Liquid temperature } \\
T_{\text {bulk }}\left({ }^{\circ} \mathrm{C}\right)\end{array}$ & $\begin{array}{c}\text { Subcooling } \\
\Delta T_{\text {sub }}\left({ }^{\circ} \mathrm{C}\right)\end{array}$ \\
\hline CEBF & $P(\mathrm{kPa})$ & 22.1 & 25.5 \\
SE & 101.2 & 21.3 & 26.2 \\
CEAF & 101.0 & 20.5 & 27.1 \\
\hline
\end{tabular}

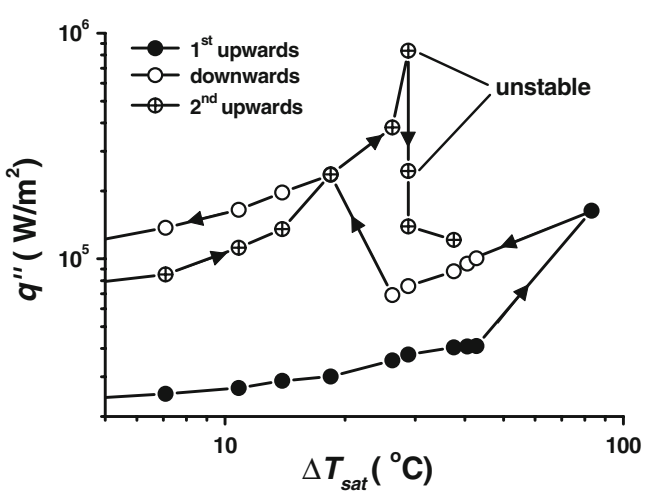

Fig. 2 Heat transfer curves in microgravity

The space experiment was carried out aboard the 22nd recoverable satellite of China in September, 2005. The level of residual gravity is estimated as $10^{-3} \sim 10^{-5} g_{0}$. Before and after the space flight, a series of control experiments using the same facility were also conducted. The space experiment and the control experiments are performed at $0.1 \mathrm{MPa}$ and with a liquid subcooling of $26^{\circ} \mathrm{C}$ nominally. The detailed conditions are listed in the Table 1, where "SE" denotes the space experiment, while "CEBF" and "CEAF" denote the control experiment before and after the space flight, respectively. The variation of the pressure of the bulk liquid is no more than $3 \%$ throughout the experiments, and the variation of the temperature is no more than $1.1^{\circ} \mathrm{C}$.

\section{Results and Discussions}

Figure 2 shows the whole curve obtained in the space experiment. Similar curves can also be obtained in normal gravity, which aren't shown here. The first heat transfer mode is single-phase natural convection both in normal gravity and in microgravity. The comparison between experimental data and the prediction of commonly used correlation of Kuehn and Goldstein (1976) is shown in Fig. 3. An agreement is quite evident in normal gravity,

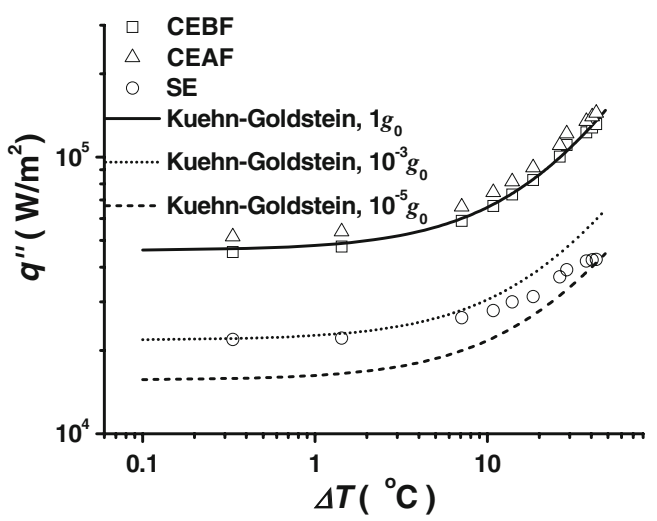

Fig. 3 Single phase natural convection both in normal and microgravity 
which warrants reasonable confidence in the data. Comparing the results of space experiments with the correlation's predictions, it's indicated that the residual gravity during the space experiment is in the range of $10^{-3} \sim 10^{-5} g_{0}$, which is equivalent to that given by the satellite establishment.

The onset of boiling occurs at the 16th step as two-mode transition boiling both in the space experiment and in ground experiments. The sequence of pictures in Fig. 4 (for magnitude estimation, the distance between the two copper poles is $30 \mathrm{~mm}$ ) corresponds to the onset of boiling in microgravity. About $0.12 \mathrm{~s}$ after the beginning of the 16th step, a large amount of vapor springs out throughout the wire with a very irregular interface. Due to the surface tension, a large spherical bubble is eventually formed. It stays on the wire continuously without enclosing the wire in the subsequent experiments, and coalesces continually up adjacent small bubbles. The boiling reached a steady state in about $3 \mathrm{~s}$ after the beginning of the 16th step. The superheat of the onset of boiling is $83^{\circ} \mathrm{C}$, which is independent, or at least, dependent much weakly on gravity. It ought to be pointed out that the actual superheat of the onset of boiling will locate in the range between the 15th and 16th set-point, or $42^{\circ} \mathrm{C}$ to $83^{\circ} \mathrm{C}$, due to the limitation of the present control method. The heat flux, however, decreased in microgravity to about $40 \%$ of that in normal gravity, while about $20 \%$ decrease for two-mode transition boiling was found in our preliminary experiments in the Drop Tower Beijing (Zhao et al. 2005).

Figure 5 shows the typical process of coalescence, vibration and departure of bubbles in the regime of fully developed nucleate pool boiling with high heat flux. It is found that the vibration due to coalescence of adjacent bubbles is the primary reason of bubble departure in microgravity in this regime. This is similar to the observations by Straub (2001), in which the bubble departure is attributed to surface tension effects, to "bubble ripening" and coalescence processes.

On the contrary, distinct bubble behaviors are observed in discrete bubble regime of nucleate pool boiling in microgravity (Fig. 6), comparing with those in normal gravity. Figure 7 shows the statistical data of discrete bubbles which appeared in the central section of the wire and moving in the observing plane. It is found that three critical bubble diameters, namely $D_{\mathrm{b} 1}=0.3 \mathrm{~mm}, D_{\mathrm{b} 2}=$ $3.5 \mathrm{~mm}, D_{\mathrm{b} 3}=8.4 \mathrm{~mm}$, are observed in the space experiments, which divide the bubbles into four groups. The tiny bubbles with diameter less than $0.3 \mathrm{~mm}$ are continually forming and growing on the heater surface before departing slowly from the wire when their sizes exceed some critical value $(0.3 \mathrm{~mm})$. The bigger bubbles with diameter between 3.5 and $6.6 \mathrm{~mm}$ stay on the wire, oscillate along the wire, and coalesce with adjacent bubbles. The biggest bubble, as
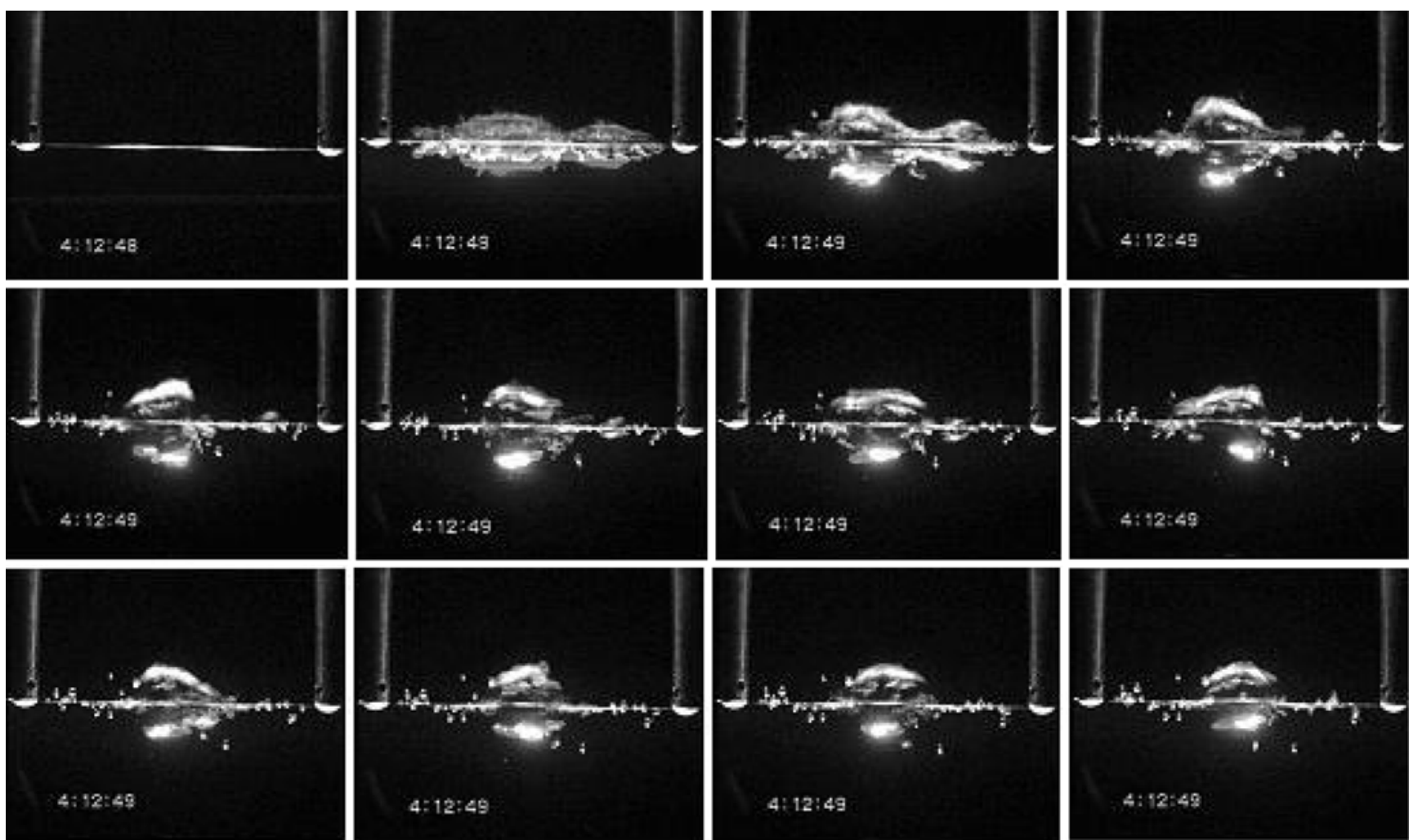

Fig. 4 A sequence of pictures of bubble development after the onset of boiling in microgravity. The time interval between sequent images is $1 / 25 \mathrm{~s}$ 
Fig. 5 Coalescence, vibration and departure of bubblers in fully developed nucleate pool boiling regime in microgravity. The time interval between sequent images is $1 / 25 \mathrm{~s}$
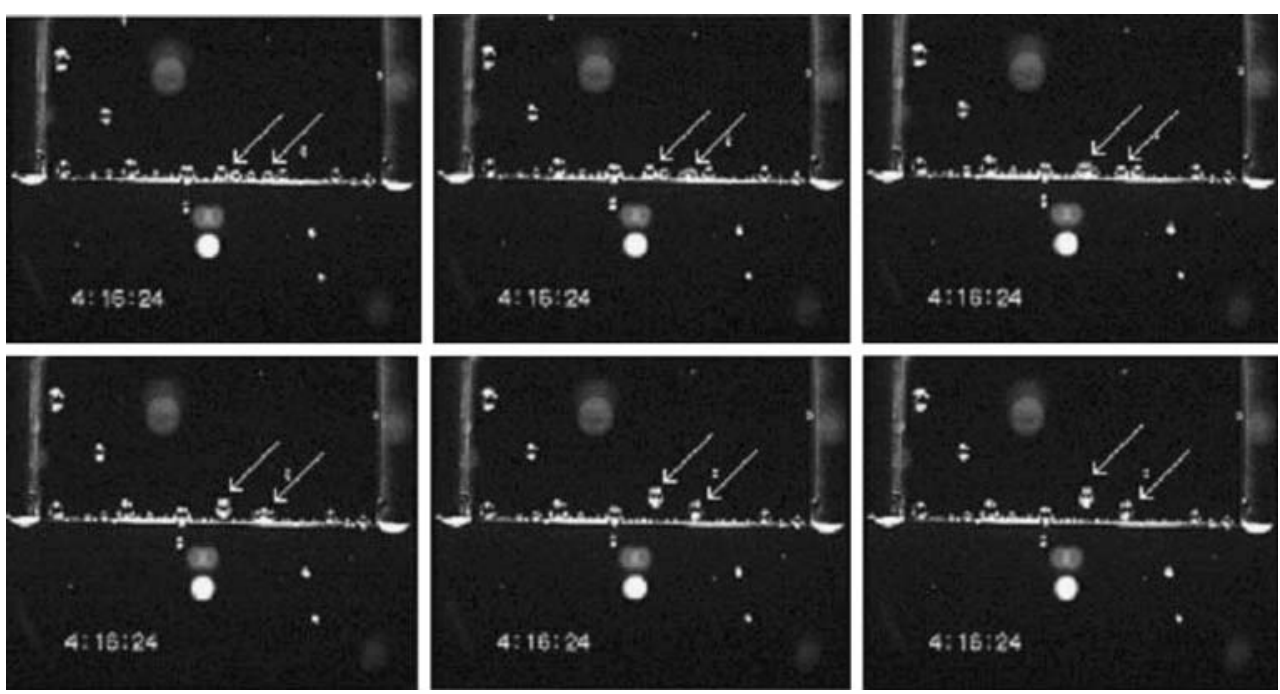

mentioned above, yielded during the onset of boiling and staying continuously on the wire, also departs from the wire when its size exceeds another critical value of about $8.4 \mathrm{~mm}$. The behaviors of the tiny bubbles are observed both in microgravity and in normal gravity, while the last two kinds of bubble behaviors are observed only in microgravity aboard the satellite. It's also observed that some bubbles move to the wire in microgravity.

Presently, no model can predict the above observation. For example, the models of Karri (1988) and Lee (1992) predict respectively the departure diameter as 0.28 and $0.13 \mathrm{~mm}$, close to the first departure diameter observed in the present study, while the models of Fritz (1934) and Zeng et al. (1993) predict respectively the departure diameter as 10.9 and $7.1 \mathrm{~mm}$, close to the second one. In all these models, the Marangoni effect is ignored.

During the process of pool boiling, the liquid temperature near the heater surface is higher than that far away from the heater surface. Therefore, a distinct temperature gradient will be formed in the liquid around the bubble. Generally, the surface tension decreases as the increase of temperature. Then the Marangoni force will pull the bubble

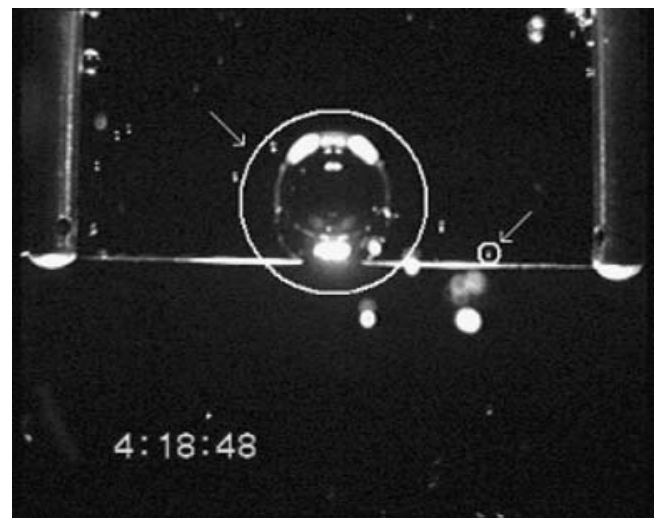

Fig. 6 Behaviors of discrete bubbles in microgravity moving towards the high temperature side (namely the heater surface), which prevents the bubble from departing from the heater's surface. So the Marangoni force $\left(F_{\mathrm{m}}\right)$ should be considered as the resistant force $\left(F_{\mathrm{R}}\right)$,

$F_{\mathrm{m}}=2 K \pi\left|\sigma_{\mathrm{T}}\right| T^{\prime} R^{2}$

Where $R, \sigma_{\mathrm{T}}$ and $T^{\prime}$ denote the bubble radius, the temperature coefficient of surface tension and the temperature gradient, respectively. The empirical coefficient $K$ is necessary for the modification of the departure from the linear theory of Young et al. (1959). In general, the value of $K$ must be determined by experiments. In the plane case, $K$ tends to about 0.3 when the Marangoni number tends to infinity (Subramanian and Balasubramaniam 2001). Further decrease may be observed in the present case due to the difference from the plane case.

Other forces acting on the bubble (Fig. 8) can be expressed according to Karri (1988) and Lee (1992). Except the Marangoni force, the buoyancy force $F_{\mathrm{b}}$, the inertia force $F_{\mathrm{i}}$, and the pressure force $F_{\mathrm{p}}$ should be considered as the departure forces $\left(F_{\mathrm{D}}\right)$ since they pull the bubble to depart from the heater surface, while the drag force $F_{\mathrm{d}}$ and the

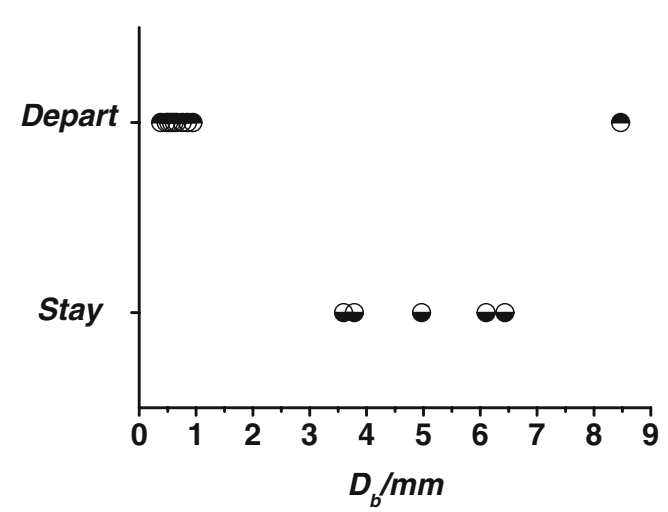

Fig. 7 Bubble departure diameters in the discrete bubble regime 


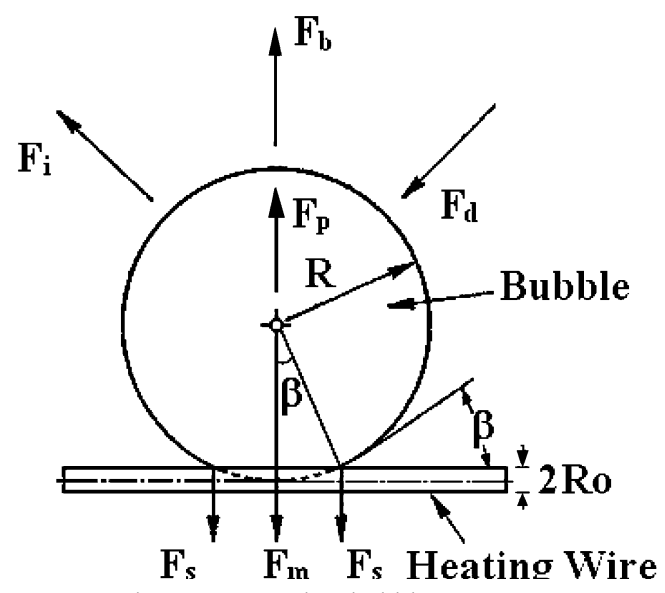

Fig. 8 Forces acting on a growing bubble

surface tension force $F_{\mathrm{s}}$ should be considered as the resistant forces $\left(F_{\mathrm{R}}\right)$ since they prevent the bubble from departing from the heater's surface. Therefore, the sum of the forces acting on the bubble is,

$$
\begin{aligned}
& F=F_{\mathrm{D}}-F_{\mathrm{R}}=\left(F_{\mathrm{i}}+F_{\mathrm{p}}+F_{\mathrm{b}}\right)-\left(F_{\mathrm{d}}+F_{s}+F_{\mathrm{m}}\right) \\
& F_{\mathrm{i}}=-\frac{4}{3} \pi R^{3} \rho_{\mathrm{L}} \frac{d^{2} R}{d \tau^{2}} \\
& F_{\mathrm{p}}=\left(\frac{2 \sigma}{R}+\frac{\rho_{\mathrm{L}} R}{3} \frac{d^{2} R}{d \tau^{2}}+C_{\mathrm{d}} \frac{\rho_{\mathrm{L}}}{8}\left(\frac{d R}{d \tau}\right)^{2}\right) A_{\mathrm{c}} \\
& F_{\mathrm{b}}=\frac{4}{3} \pi R^{3}\left(\rho_{\mathrm{L}}-\rho_{\mathrm{V}}\right) g \\
& F_{\mathrm{d}}=C_{\mathrm{d}} \frac{\rho_{\mathrm{L}}}{2}\left(\frac{d R}{d \tau}\right)^{2} A_{\mathrm{c}} \\
& A_{\mathrm{c}}=4 R R_{0} \sin ^{2} \beta \\
& C_{\mathrm{d}}=5,360\left(\frac{\rho_{\mathrm{L}} R}{\mu_{\mathrm{L}}} \frac{d R}{d \tau}\right)^{-0.79} \\
& F_{0} \sigma \sin ^{2} \beta
\end{aligned}
$$

there, $g, R, R_{0}, \beta, \mu, \rho$, and $\tau$ denote the residual gravity, bubble radius, wire radius, contact angle, dynamic viscosity, density, and time, respectively. The subscripts $\mathrm{L}$ and $\mathrm{V}$ denote the liquid and vapor phases. According to Karri (1988) and Lee (1992), an asymptotic bubble growth relation can be assumed, namely $R=E \tau^{1 / 2}, E=\frac{1}{2 \sqrt{\pi}} J a \times$ $\sqrt{\alpha_{\mathrm{L}}}$, where, Ja and $\alpha_{\mathrm{L}}$ denote the Jacob number and the heat diffusivity coefficient of the liquid, respectively. Thus, the formula (2) can be rewritten as

$f(y)=C_{4} y^{4}+C_{3} y^{3}+C_{1} y+C_{0}$

where,

$y=\tau^{1 / 2}$

$C_{0}=R_{0} E^{3} \rho_{\mathrm{L}} \sin ^{2} \beta\left(\frac{1}{3}-\frac{3}{8} C_{\mathrm{d}}\right)$

$C_{1}=4 \sigma R_{0} \sin ^{2} \beta+\frac{\pi}{3} \rho_{\mathrm{L}} E^{4}$

$C_{3}=-2 K \pi\left|\sigma_{\mathrm{T}}\right| T^{\prime} E^{2}$

$C_{4}=\frac{4}{3} \pi E^{3}\left(\rho_{\mathrm{L}}-\rho_{\mathrm{V}}\right) g$

According to Eq. 2, the following conclusion can be obtained: If $f(y)<0$, the departure force is smaller than the resistant force, so the bubble will stay on the heater; if $f(y)>0$, the departure force is larger than the resistant force and the bubble will depart from the heater.

Using the following parameters for R113, $p=0.1 \mathrm{MPa}$, $\Delta T_{\text {sub }}=26^{\circ} \mathrm{C}, \Delta T_{\text {sat }}=20^{\circ} \mathrm{C}, \beta=5^{\circ}, K=0.035$, the trends of $f$ (y) with gravity are plotted in Fig. 9. Here the temperature gradient is calculated as $T^{\prime}=\left(T_{\mathrm{W}}-T_{\text {bulk }}\right) / L$, and half of the boiling chamber width is used for the characteristic length $L$. It implies that the temperature distribution along the bubble surface is not affected by the phase change, which is similar with Shatto and Peterson (1999) but different from Straub (2001).

Considering the positive half plane of $D_{\mathrm{b}}$, there is only one intersection between Eq. 10 and the abscissa, i.e. $D_{\mathrm{b} 1} \approx$

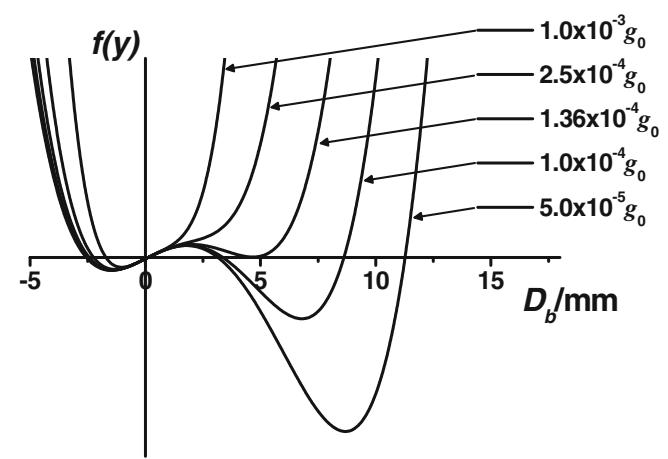

Fig. 9 Trend of $f(y)$ with gravity 
$0.11 \mathrm{~mm}$, in normal gravity. It is very close to that predicted by Lee's model, indicating that the effect of Marangoni convection can be ignored in normal gravity. The value of $D_{\mathrm{b} 1}$ is gradually increasing as the residual gravity decreasing, although the increment is very small. On the contrary, when the gravity reduces to $1.36 \times 10^{-4} \mathrm{~g}$, a second intersection $\left(D_{\mathrm{b} 2}\right)$ appears. With further decrease of the residual gravity, there will be three intersections $\left(D_{\mathrm{b} 1}, D_{\mathrm{b} 2}\right.$, and $D_{\mathrm{b} 3}$ ), which divide the positive abscissa into four regions:

$1 \quad D_{\mathrm{b}}<D_{\mathrm{b} 1}, f(y)<0$, the bubble stays on the heater surface;

$2 D_{\mathrm{b} 1}<D_{\mathrm{b}}<D_{\mathrm{b} 2}, f(y)>0$, the bubble departs from the heater surface;

$3 D_{\mathrm{b} 2}<D_{\mathrm{b}}<D_{\mathrm{b} 3}, f(y)<0$, the bubble stays on the heater surface; and

$4 \quad D_{\mathrm{b}}>D_{\mathrm{b} 3}, f(y)>0$, the bubble departs from the heater surface.

It agrees qualitatively with the observation in space experiment. Furthermore, a quantitative agreement can also be obtained with $K=0.035$, in which $D_{\mathrm{b} 1}=0.13 \mathrm{~mm}, D_{\mathrm{b} 2}=$ $3.4 \mathrm{~mm}, D_{\mathrm{b} 3}=8.6 \mathrm{~mm}$ are predicted if the residual gravity is assumed as $10^{-4} g_{0}$ in the space experiments. Here, it ought to be pointed out that the determination of the parameter $K$ is also dependent on the actual value of the residual gravity.

\section{Summary}

A temperature-controlled pool boiling (TCPB) device has been developed to study the bubble behavior and heat transfer in pool boiling phenomenon both in normal gravity and in microgravity. The space experiment has been performed aboard the 22nd Chinese recoverable satellite, while the ground experiments have also been conducted before and after the flight. In this paper, only dynamic behaviors of vapor bubbles observed in these experiments are reported and analyzed.

In the regime of fully developed nucleate boiling, the interface oscillation due to coalescence of adjacent tiny bubbles is the primary reason of the departure of bubbles in microgravity. On the contrary, it's observed in the discrete bubble regime that there exist three critical bubble diameters in microgravity, dividing the whole range of the observed bubbles into four regimes. Firstly, tiny bubbles are continually forming and growing on the heating surface before departing slowly from the wire when their sizes exceed some value of the order of $10^{-1} \mathrm{~mm}$. The bigger bubbles with about several millimeters in diameter stay on the wire, oscillate along the wire, and coalesce with adjacent bubbles. The biggest bubble formed immediately after the onset of boiling stays continuously on the wire and swallows continually up adjacent small bubbles until its size exceeds another critical value. The same behavior of tiny bubbles can also be observed in normal gravity, while the others are observed only in microgravity. Considering the Marangoni effect, a mechanistic model about bubble departure is presented to reveal the mechanism underlying this phenomenon. The predictions are qualitatively consistent with the experimental observations.

Acknowledgements This study is supported financially by the Chinese Academy of Sciences under the grant of KJCX2-SW-L05, and the National Natural Science Foundation of China under the grant of 10432060. The authors also wish to acknowledge the fruitful discussion with Prof. H. Ohta (Kyushu University, Japan).

\section{References}

Di Marco, P.: Review of reduced gravity boiling heat transfer: European research. J. Jpn. Microgravity Appl. 20, 252-263 (2003)

Di Marco, P., Grassi, W.: Motivation and results of a long-term research on pool boiling heat transfer in low gravity. Int. J. Therm. Sci. 41, 567-585 (2002)

Di Marco, P., Grassi, W., Trentavizi, F.: Pool film boiling experiments on a wire in low gravity: preliminary results. In: Microgravity Transport Processes in Fluid, Thermal, Biological \& Material Sciences. Banff, Alberta, Canada, Sep. 30-Oct. 5 (2001)

Fritz, W.: Berechnungen des Maximalvolumens von Dampfblasen. Z. Phys. 36, 379-384 (1934)

Kim, J.: Review of reduced gravity boiling heat transfer: US research. J. Jpn. Microgravity Appl. 20, 264-271 (2003)

Kuehn, T.H., Goldstein, R.J.: Correlating equations for natural convection heat transfer between horizontal circular cylinders. Int. J. Heat Mass Transfer 19, 1127-1134 (1976)

Lee, D.J.: Bubble departure radius under microgravity. Chem. Eng. Commun. 117, 175-189 (1992)

Lienhard, J.H., Dhir, V.K.: Hydrodynamic prediction of peak pool boiling heat fluxes from finite bodies. J. Heat Transfer, Trans. ASME 95, 152-158 (1973)

McGillis, W.R., Carey, V.P.: On the roles of Marangoni effects on the critical heat flux for pool boiling of binary mixtures. J. Heat Transfer, Trans. ASME 118, 103-109 (1996)

Motoya, D., Haze, I., Osakabe, M.: Effect of fouling on nucleate pool boiling in microgravity and earth gravity, In: Proc. ASME HTD vol. 364-1, pp. 303-310, (1999)

Ohta, H.: Review of reduced gravity boiling heat transfer: Japanese research. J. Jpn. Microgravity Appl. 20, 272-285 (2003)

Reddy Karri, S.B.: Dynamics of bubble departure in micro-gravity. Chem. Eng. Commun. 70, 127-135 (1988)

Rule, T.D., Kim, J.: Heat transfer behavior on small horizontal heaters during pool boiling of FC-72. J. Heat Transfer 121, 386-393 (1999)

Shatto, D.P., Peterson, G.P.: Pool boiling critical heat flux in reduced gravity. J. Heat Transfer, Trans. ASME 121, 865-873 (1999)

Siegel, R., Usiskin, C.: Photographic study of boiling in absence of gravity. J. Heat Transfer Trans. ASME 81C, 230-236 (1959)

Sitter, J.S., Snyder, T.J., Chung, J.N., Marston, P.L.: Acoustic field interaction with a boiling system under terrestrial gravity and microgravity. J. Acoust. Soc. Am. 104, 2561-2569 (1998)

Steinbichler, M., Micko, S., Straub, J.: Nucleate boiling heat transfer on small hemispherical heaters and a wire under microgravity. In: Heat Transfer 1998, Proc. 11th IHTC, Kyongju, Korea, vol. 2, pp. 539-544 (1998) 
Straub, J.: Boiling heat transfer and bubble dynamics in microgravity, Adv. Heat Transfer. 35, 57-172 (2001)

Straub, J., Micko, S.: Boiling on a wire under microgravity conditionsfirst results from a space experiment, performed in May 1996. In: Proc. Eurotherm. Seminar No. 48, Paderbom, Germany (1996)

Straub, J., Zell, M., Vogel, B.: Boiling under microgravity conditions. In: Proc. 1st European Symp. Fluids in Space, Ajaccio, France, 1991. ESA SP-353 (1992)

Subramanian, R.S., Balasubramaniam, R.: The motion of bubbles and drops in reduced gravity. Cambridge University Press, Cambridge, UK (2001)

Tokura, I., Hanaoka, Y., Suzuki, H., Hirata, H., Yoneta, M.: Boiling heat transfer on thin wires in a micro-gravity field. In: Proc.
ASME/JSME Thermal Eng. Conf., Maui, USA, v.4, pp. 555-560 (1995)

Wan, S.X., Zhao, J.F., Liu, G., Hu, W.R.: TCPB device: description and preliminary ground experimental results. In: 54th Int. Astronautical Cong., Bremen, Germany, Sep. 29-Oct 3 (2003)

Young, N.O., Goldstein, J.S., Block, M.J.: The motion of bubbles in a vertical temperature gradient. J. Fluid Mech. 6, 350-356 (1959)

Zeng, L.Z., Klausner, J.F., Mei, R.: A unified model for the prediction of bubble detachment diameters in boiling system-1. pool boiling. Int. J. Heat Mass Transfer 36, 2261-2270 (1993)

Zhao, J.F., Wan, S.X., Liu, G., Hu, W.R.: Experimental study on subcooled pool boiling in microgravity utilizing Drop Tower Beijing/NMLC. Proc. ISMF'05, Xi'an, China, 3-6 July (2005) 